\title{
Dimensionality of hypercube clusters
}

\author{
Forrest H. Kaatz, Mesalands Community College, Tucumcari, NM, USA \\ Adhemar Bultheel, Dept. Computer Sci., KU Leuven, Heverlee, Belgium
}

\begin{abstract}
We investigate clusters of hypercubes in $d$-dimensional space as a function of the number of vertices, $N$, and number of cluster shells, $L$. The number of links, vertices, and exterior vertices exhibit 'magic number' characteristics versus $L$, as the dimension of the space changes. Starting with only the spatial coordinates, we create an adjacency and distance matrix that facilitates the calculation of topological indices, including the Wiener, hyper-Wiener, reverse Wiener, Szeged, Balaban, and Kirchhoff indices. Some known topological formulas for hypercubes when $L=1$ are experimentally verified. The asymptotic limits with $N$ of the topological indices are shown to exhibit power law behavior whose exponent changes with $d$ and type of topological index. The asymptotic graph energy is linear with $N$, whose slope changes with $d$, and in 2D agrees numerically with previous calculations. Also, the thermodynamic properties such as entropy, free energy, and enthalpy of these lattices show logarithmic behavior with increasing $N$. The hypercubic clusters are projected onto 3D space when the dimensionality $d>3$.
\end{abstract}

\section{Introduction}

A $d$-dimensional hypercube $Q_{d}$ can be defined recursively in terms of two graphs and a Cartesian product as

$$
Q_{d}= \begin{cases}Q_{0}, & d=0 \\ K_{2} \times Q_{d-1}, & d>0\end{cases}
$$

where $Q_{0}$ is a trivial graph having one node, $K_{2}$ is a complete graph with two nodes, and $\times$ represents a Cartesian product $[1,2]$. Thus, the $d$-cube, $Q_{d}$, is a graph with $2^{d}$ Boolean vectors with coordinates 0 or 1 , where two nodes are adjacent if they differ in exactly one coordinate. One application of hypercubes is for multicore computer architectures with a publication history of over 30 years $[2,3]$. Our interest is in the graph properties, which also have a long history [1].

Clusters have been considered in geometry [4] and in 3D nanoscience [5], but we are not aware of previous work with hypercubes. A large collection of results from metal nanoclusters exists, as metals comprise $75 \%$ of elements in the Periodic Table. These results are fairly 
recent with the majority occurring since the beginning of this century 15 years ago from developments in chemical synthesis [6]. Semiconductor nanocrystals or quantum dots are also of interest as they have novel properties due to doping [7].

Topological indices can be traced back to the Wiener index in 1947 [8], although it was some time later in 1971 [9] that its status in the field of mathematical chemistry became established. Today there are many topological indices, and there have been fluctuations in the number of publications on these topics, with some indices becoming more relevant in the lexicon of topological chemistry. Recently, a $\mathrm{C}++$ program detailing the numerical calculation of many of these indices has been made available [10] and we have rewritten these programs with a MATLAB program that calculates the adjacency and distance matrices from only the spatial coordinates. It is worthwhile to note that the $\mathrm{C}++$ program only works for fullerenes, while our program only requires the coordinates. In a different type of analysis, we have modeled both fullerenes and nanoclusters [11,12].

Asymptotic limits have been studied for the Wiener index [13]. The current authors also looked at limits for topological indices in 3D for nanoclusters [12]. Topological indices have power law large $N$ behavior, where the exponent depends on the dimension, $d[12,13]$. In this manuscript, we examine in more detail these relationships for several topological indices as the dimensionality changes. We also examine the asymptotic limit of the graph energy, which has been examined in detail for $d=2$ [14].

In the last 15 years, complex network theory has evolved to consider the statistical mechanics of graphs [15,16]. Examples of networks include the cell, a network of chemicals linked by chemical reactions, and the Internet, which has physical connections. More recent analysis has considered the adjacency matrix as a central element of the partition function, with thermodynamic definitions resulting from this designation [17]. We consider the large $\mathrm{N}$ asymptotic behavior of the subsequently defined entropy, free energy, and enthalpy as it applies to clusters of hypercubes.

\section{Methods}

As mentioned, we use a theoretical graph - network approach where an adjacency matrix is created which contains a 1 at position $(i, j)$ if vertices $i, j$ are nearest neighbors. Thus,

$$
\mathbf{A}(i, j)= \begin{cases}1, & \text { if } i, j \text { are n.n. } \\ 0, & \text { otherwise }\end{cases}
$$

Some topological indices are most easily calculated from the distance matrix, which is defined as

$$
D_{i j}= \begin{cases}0, & i=j \\ d_{i j}, & i \neq j\end{cases}
$$

where $d_{i j}$ is the length of the shortest path from $i$ to $j$. An efficient algorithm for the calculation of the distance matrix from the adjacency matrix exists [18]. Using this definition, 
we can calculate the Wiener index, $W(G)$ and hyper-Wiener index, $W W(G)$ as

$$
\begin{aligned}
& W(G)=\frac{1}{2} \sum_{i=1}^{n} \sum_{j=1}^{n} d_{i j}=\sum_{i>j=1}^{n} d_{i j} \\
& W W(G)=\frac{1}{2} \sum_{i=1}^{n} \sum_{j=1}^{n}\left(d_{i j}+d_{i j}^{2}\right)
\end{aligned}
$$

and the reverse Wiener index $[19] \Lambda(G)$ is

$$
\Lambda(G)=r W=n(n-1) D / 2-W(G)
$$

where $D$ is the topological diameter defined as

$$
D=\max _{i}\left\{\max _{j}\left(D_{i j}\right)\right\}
$$

and $D_{i j}$ is an element of the distance matrix. A related index is the Szeged index introduced by Gutman [20], where

$$
S z(G)=\sum_{\text {edges }} n_{i} n_{j}
$$

where, for any edge $i j, n_{i}$ counts the vertices of $G$ that are closer to vertex $i$ than to $j$, and $n_{j}$ counts those that are closer to $j$ than to $i$, with vertices equidistant from $i$ and $j$ ignored. This is a natural generalization of the Wiener index since (5) is actually the formula used by Wiener for acyclic graphs (for which $W=S z$ ) [21]. The Szeged index also has some other similarities to the Wiener index, in that if $G$ and $G^{\prime}$ are catacondensed benzenoid systems with an equal number of hexagons, then $W(G)=W\left(G^{\prime}\right)(\bmod 8)$ and $S z(G)=S z\left(G^{\prime}\right)(\bmod 8)$ [22]. Also for any connected network $G, S z(G) \geq W(G)$ [23].

Another invariant derived from the distance matrix is the Balaban index [24-26], $J$,

$$
J(G)=\frac{b}{c+1} \sum_{\text {edges }}\left(d_{i} d_{j}\right)^{-1 / 2}
$$

where $b$ is the number of edges, $c$ is the number of primitive cycles and $d_{i}$ is the row sum

$$
d_{i}=\sum_{j=1}^{n} d_{i j}
$$

The Balaban index was originally introduced as being less degenerate than other indices [24], and has some asymptotic limits: for a linear alkane $C_{n} H_{2 n+2}$ it tends to $\pi$, while for highly branched alkanes, $J$ increases without limit.

The Kirchhoff index can be determined from the Laplacian matrix, where $\mathbf{L}$ is defined as

$$
\mathbf{L}=\Delta-\mathbf{A}
$$


and $\Delta$ is a diagonal matrix of order $n$, with diagonal elements $=$ the number of first neighbors, or the number of non-zero entries in a column in the adjacency matrix, $\mathbf{A}$ [27]. Then the Kirchhoff index is

$$
K f(G)=n \sum_{i=1}^{n-1} \frac{1}{\lambda_{i}}
$$

and $\lambda_{i}$ is an eigenvalue of $\mathbf{L}$.

The complex systems network theory uses the adjacency matrix in the model [17], where the partition function is

$$
Z=\operatorname{Tr} \mathrm{e}^{\beta \mathbf{A}}
$$

and $\beta=1 / k_{B} T$ is the inverse temperature, with $k_{B}$ Boltzmann's constant. For an unweighted network, $\beta=1$, which is what we use. The statistical mechanics quantities are then calculated from the probability of occupying a state $j$ as

$$
p_{j}=\frac{e^{\beta \lambda_{j}}}{Z}
$$

where $\lambda_{j}$ is an eigenvalue of $\mathbf{A}$. The informational Shannon entropy is then

$$
S(G, \beta)=-\sum_{j} p_{j} \ln p_{j}
$$

for a graph $G$ [28]. The total energy $H$, or enthalpy, and Helmholtz free energy, $F$, are related by $F=H-T S$, which results in the expressions

$$
F(G, \beta)=-\frac{1}{\beta} \ln Z
$$

and

$$
H(G, \beta)=-\frac{1}{Z} \operatorname{Tr}\left(\mathbf{A} \mathrm{e}^{\beta \mathbf{A}}\right)
$$

where $\mathbf{A}$ is again the adjacency matrix [17].

There has been previous work on the asymptotic behavior of lattices. The large $N$ behavior of the topological indices follows a power law when $d=3[12]$. For the Wiener index, there exists a conjecture [13] that

$$
\lim _{N \rightarrow \infty} W(N)=a N^{s}, \quad s=2+1 / d
$$

The definition of lattice energy for a graph is

$$
E(G)=\sum_{i=1}^{N}\left|\lambda_{i}(G)\right|
$$

where $\lambda_{i}(G)$ is an eigenvalue of $\mathbf{A}[14]$. In 2D, there exists a formula for the asymptotic energy, which is $[14]$

$$
\lim _{N \rightarrow \infty} E(G)=1.6211 N
$$




\begin{tabular}{|l|c|c|c|c|}
\hline \multicolumn{5}{|c|}{ Magic formulas } \\
\hline Dimension & $d=4$ & $d=3$ & $d=2$ & $d=1$ \\
\hline Vertices & $(2 L)^{4}$ & $(2 L)^{3}$ & $(2 L)^{2}$ & $2 L$ \\
\hline Links & $32 L^{3}(2 L-1)$ & $12 L^{2}(2 L-1)$ & $4 L(2 L-1)$ & $2 L-1$ \\
\hline Exterior Vertices & $16\left(4 L^{3}-6 L^{2}+4 L-1\right)$ & $8\left(3 L^{2}-3 L+1\right)$ & $4(2 L-1)$ & 2 \\
\hline
\end{tabular}

Table 1: Magic formulas for clusters of hypercubes when $d \leq 4$.

Our work adds to this analysis by adding more topological indices and more lattice dimensions when considering the graph energy. Also, when using a Hamiltonian in the statistical mechanics approach, we have demonstrated large $N$ logarithmic behavior for the thermodynamic properties of 3D nanoclusters [11]. This work extends that analysis as the dimensionality changes and we find the large $\mathrm{N}$ logarithmic behavior still applies.

\section{Results}

We construct clusters in $d$-dimensional space where each layer sequentially covers the previous one. When considering clusters in geometry, one can derive 'magic formulas' for the number of vertices, links and exterior vertices. Some work has been done in this regard for $2 \mathrm{D}$ and $3 \mathrm{D}$ clusters [4]. Our work considers clusters in $d$-dimensional space. Table 1 shows magic formulas for clusters with $d \leq 4$.

Thus, our hypercube clusters have $N=(2 L)^{d}$ vertices, where $L$ is the number of cluster layers. Figure 1 shows clusters for $L=1,2$ and $d \leq 4$. We project the $d=4$ dimensional hypercube onto the diagonal 3D subspace containing the center of the hypercube and the vertices $(0,0,0,0),(0,0,1,1)$, and $(0,1,0,1)$. This is the diagonal $3 \mathrm{D}$ subspace through the center of the hypercube and spanned by the orthogonal vectors $(1,1,1,1),(1,1,-1,-1)$, and $(1,-1,1,-1)$. In general for $d \geq 4$ we take a $3 \mathrm{D}$ subspace containing the center of the hypercube and three vertices such that the three vectors connecting the center with these three vertices are 'as orthogonal as possible'. This is the affine subspace through the center of the hypercube and spanned by the vector $(1,1, \ldots 1)$ and two other vectors with \pm 1 coordinates such that the absolute value of their inner product is minimal.

We calculate the six topological indices from a MATLAB program. We have previously demonstrated that this program reproduces the values associated with $C_{60}$ for all six indices, so we are confident of our calculations [12]. Figure 2 shows the behavior of the indices for a fixed $N=4096$, where $d$ and $L$ changes to accommodate that $N$. The Szeged index is monotonically increasing, while the Balaban index is relatively high for $d=1$, since that is an acyclic graph and $J \rightarrow \pi$, but for $d=2, J$ decreases due to the cycles in the graph. All the other indices are monotonically decreasing with a smaller decrease after $d=6$.

Formulas for the topological indices of hypercubes, $Q_{d}$, have been derived in previous publications [29-32]. For convenience, we list them in Table 2. These formulas are strictly 
A
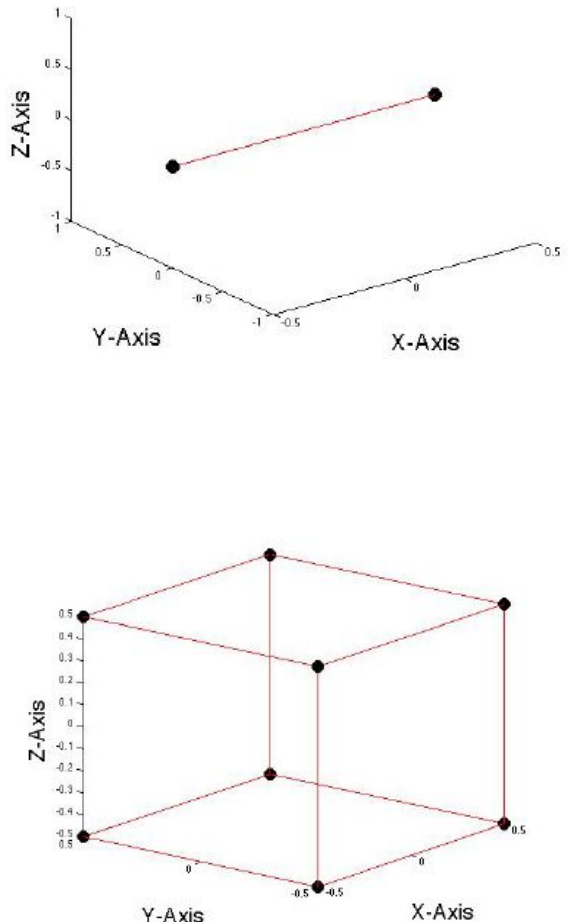

C
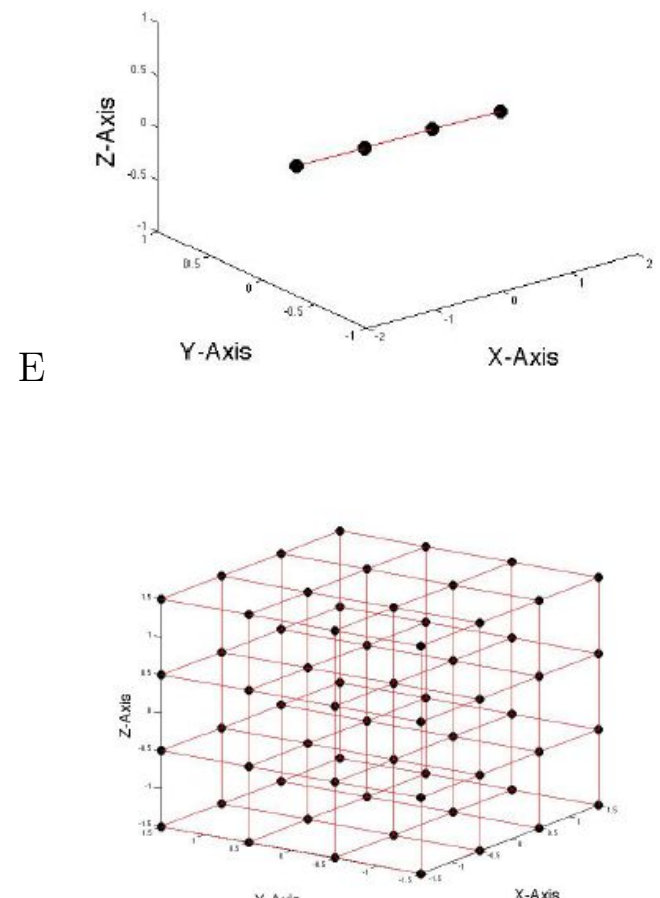

G

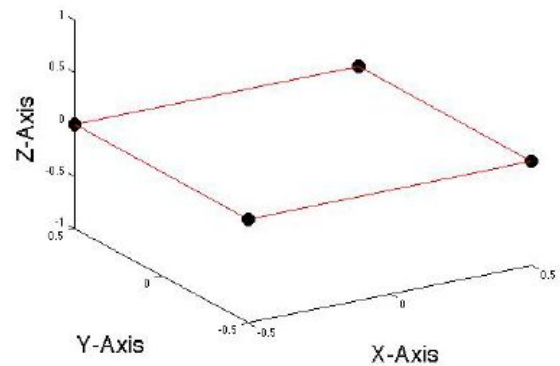

$\mathrm{B}$

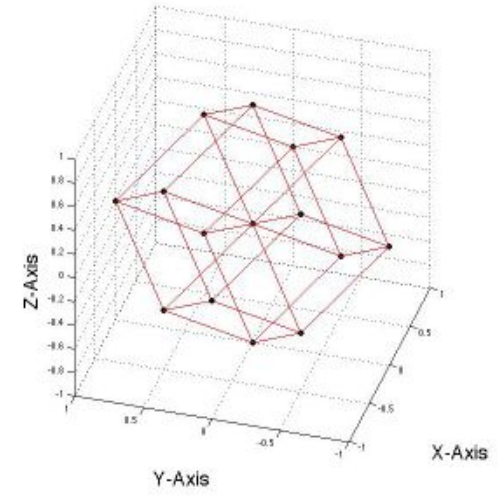

$\mathrm{D}$

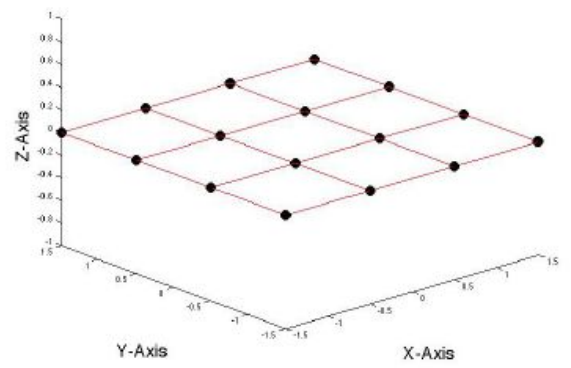

F

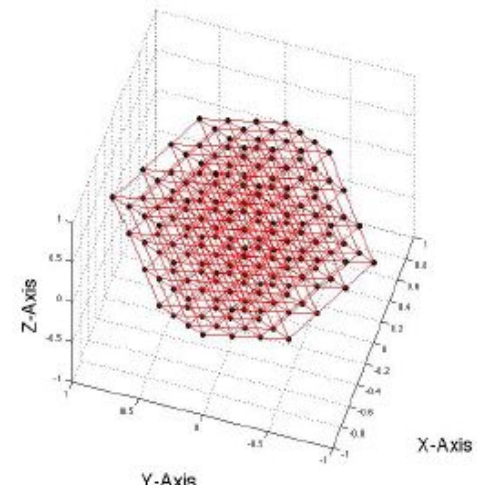

$\mathrm{H}$

Figure 1: A-D are plots of $L=1$ and $d \leq 4$. E-H are plots of $L=2$ and $d \leq 4$. For $d=4$, we have projected the hypercubic cluster onto 3D space. 


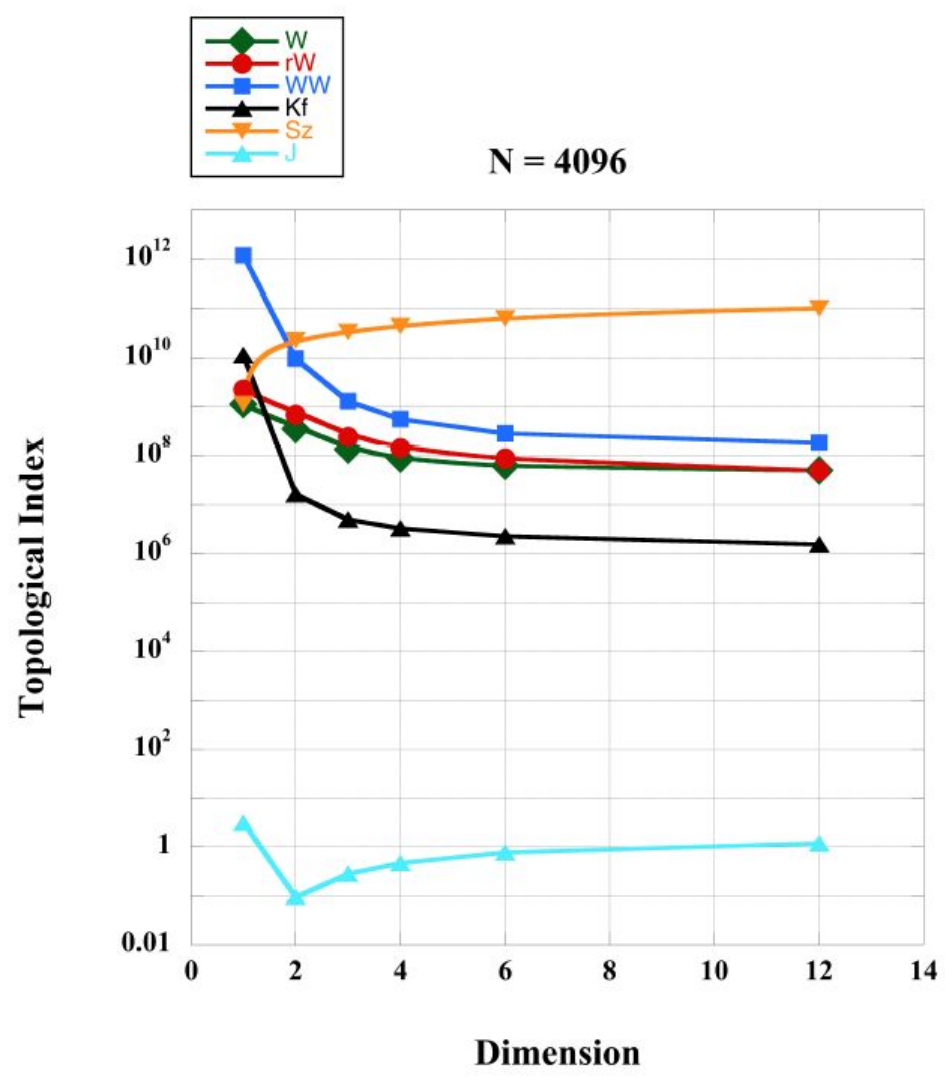

Figure 2: Line graphs of six topological indices when $N=4096$, and is fixed, as $d$ changes.

\begin{tabular}{|c|ll|}
\hline Topological index & \multicolumn{2}{|c|}{ Hypercube formula $L=1$} \\
\hline Wiener & $W(Q-d)=2^{2(d-1)} d$ & {$[29]$} \\
\hline Szeged & $S z\left(Q_{d}\right)=2^{3(d-1)} d$ & {$[30]$} \\
\hline Balaban & $J\left(Q_{d}\right)=\frac{2^{d-1} d}{2^{d-1}(d-2)+2}$ & {$[31]$} \\
\hline Kirchoff & $K f\left(Q_{d}\right)=2^{d} \sum_{i=1}^{2^{d}-1} 1 / \lambda_{i}$ & {$[32]$} \\
\hline
\end{tabular}

Table 2: Published topological formulas for hypercubes of four indices. The formula is found in the reference listed in each equation. 


\begin{tabular}{|c|c|c|c|c|c|c|c|}
\hline Index & $d=1$ & $d=2$ & $d=3$ & $d=4$ & $d=5$ & $d=6$ & $d=12$ \\
\hline Wiener & 1 & 8 & 48 & 256 & 1280 & 6144 & 50331648 \\
\hline Szeged & 1 & 16 & 192 & 2048 & 20480 & 196608 & 103079215104 \\
\hline Balaban & 1 & 2 & 2 & 1.778 & 1.599 & 1.4769 & 1.1998828239 \\
\hline Kirchoff & 1 & 5.00 & 19.33 & 68.66 & 236.53 & 809.06 & 1560002.3088 \\
\hline
\end{tabular}

Table 3: Calculated topological indices corresponding to the formulas listed in Table 2.

for $L=1$ in our designation of cluster geometry. We mention here that a different formula for the Szeged index is listed in [33] as

$$
S z\left(Q_{d}\right)=2^{3 d-2} .
$$

According to our calculations, this is incorrect, and we agree with the formula listed in Table 2. The results of our calculated values of the four indices for the $L=1$ hypercubes are shown in Table 3. These values agree with the formulas in Table 2.

We note that the formula for the Kirchhoff index can be simply obtained from the definition after replacing $N$ with $2^{d}$. However, a computer best calculates the eigenvalues of the Laplacian used in the Kirchhoff index.

The asymptotic behavior of the indices for different dimensions is demonstrated in Table 4 . These limits are obtained by choosing $d$ and $L$ so that $N$ is about 30,000 for most of the calculations. These structures take about 24 hours on a computer with an i7 processor, 32GB of RAM, and running MATLAB_R2014a. It takes less time when the adjacency matrix is sparse, as when $d=1$. We find upon closer analysis, the following power law limits for large $N$

$$
\lim _{N \rightarrow \infty} T I(N)=a N^{s} .
$$

In Table 4, both a and s can be numerically interpolated from the data, using adjacent ordered pairs of $(T I, N)$, where $T I$ is a topological index as $N \rightarrow \infty$. This gives us the following results for the dependence of $s$ on $d$ for the indices

$$
\begin{gathered}
s_{W}=s_{r W}=2+1 / d, \quad d \geq 1, \quad s_{W W}=\frac{2(d+1)}{d}, \quad d \geq 1, \\
s_{S z}=3, \quad s_{K f}=\left\{\begin{array}{ll}
3, & d=1 \\
2, & d \geq 2
\end{array}, \quad s_{J}=-\frac{1}{d}, \quad d \geq 2 .\right.
\end{gathered}
$$

It appears that the Wiener and reverse Wiener indices have the same dimensional power law dependence with $2<s \leq 3$, with 2 being a lower limit as $d$ increases. For the hyper-Wiener index, we have $2<s_{W W} \leq 4$, and 2 is again a lower limit. The Szeged index is the only one that does not depend on d, with $s_{S z}=3$ for all dimensions. The Kirchhoff index is 3 for $d=1$ and is 2 for all higher dimensions. For $d=1$, the Balaban index tends to $\pi$, since there are no cycles in the straight-line graph. For higher dimensions, $d$ varies as the inverse of $d$. We comment that for $d=1$ and $d=2, W(N)$ is known exactly as [13]

$$
W(N)=\frac{1}{6}\left(N^{3}-N\right), \quad d=1 ; \quad W(N)=\frac{1}{3}\left(N^{5 / 2}-N^{3 / 3}\right), \quad d=2 .
$$




\begin{tabular}{|c|c|c|c|c|c|c|c|}
\hline$d$ & $W$ & $W W$ & $r W$ & $S z$ & $K f$ & $J$ & $E$ \\
\hline 1 & $0.16667 N^{3}$ & $0.040156 N^{4.0038}$ & $0.33293 N^{3.0001}$ & $0.16667 N^{3}$ & $0.16659 N^{3}$ & $\pi$ & $1.2734 N$ \\
\hline 2 & $0.32839 N^{2.5015}$ & $0.16342 N^{2.9848}$ & $0.62898 N^{2.5048}$ & $0.33319 N^{3}$ & $0.55871 N^{2.0696}$ & $6.1926 N^{-0.50059}$ & $1.619 N$ \\
\hline 3 & $0.49568 N^{2.334}$ & $0.3388 N^{2.6543}$ & $0.76246 N^{2.355}$ & $0.49541 N^{3.0008}$ & $0.36119 N^{1.9755}$ & $4.3716 N^{-0.32994}$ & $1.9808 N$ \\
\hline 4 & $0.62541 N^{2.2558}$ & $0.56903 N^{2.4913}$ & $0.8088 N^{2.2872}$ & $0.63481 N^{3.0042}$ & $0.27317 N^{1.959}$ & $3.5254 N^{-0.24021}$ & $2.2116 N$ \\
\hline 5 & $0.74986 N^{2.2086}$ & $0.79922 N^{2.3994}$ & $0.83488 N^{2.2471}$ & $0.74466 N^{3.0094}$ & $0.23146 N^{1.9521}$ & $3.0481 N^{-0.18611}$ & $2.407 N$ \\
\hline
\end{tabular}

Table 4: Asymptotic behavior of six topological indices and the graph energy. The coefficient and exponent are experimentally determined.

\begin{tabular}{|c|c|c|c|}
\hline$d$ & Entropy, $S$ & -Free energy, $-F$ & -Enthalpy, $-H$ \\
\hline 1 & $-0.45961+0.98617 \ln (N)$ & $0.6327+1.0234 \ln (N)$ & 1.3955 \\
\hline 2 & $-0.83514+0.96526 \ln (N)$ & $1.0978+1.0607 \ln (N)$ & $1.9328+0.095493 \ln (N)$ \\
\hline 3 & $-1.0707+0.92918 \ln (N)$ & $1.3532+1.1175 \ln (N)$ & $2.4239+0.18833 \ln (N)$ \\
\hline 4 & $-1.1226+0.88042 \ln (N)$ & $1.3935+1.1829 \ln (N)$ & $2.5162+0.30242 \ln (N)$ \\
\hline 5 & $-1.1985+0.84633 \ln (N)$ & $1.4942+1.2241 \ln (N)$ & $2.6927+0.37775 \ln (N)$ \\
\hline
\end{tabular}

Table 5: Asymptotic behavior of the thermodynamic properties. The signs of the free energy and enthalpy have been reversed so that they may be modeled.

These results are in agreement with our asymptotic limits. Our other results may help modeling efforts by providing the leading coefficient and exponent.

Also listed in Table 4 is the asymptotic behavior of the graph energy. This is linear in $N$ for all dimensions, with the coefficient increasing with $d$. We are aware of a calculation of the asymptotic behavior for $d=2$, where the authors determine the dependence listed in equation (19) [14]. Our value for the coefficient is 1.619, a difference of $-0.13 \%$ from the calculated value. A better asymptotic limit could be obtained using larger structures, at the cost of larger processing times.

In Table 5, we show the large $N$ asymptotic behavior of the thermodynamic quantities entropy, free energy, and enthalpy. The signs of the free energy and enthalpy have been reversed so that they may be modeled. These properties depend on $N$ as

$$
P=A+B \ln (N)
$$

where $P$ is the thermodynamic property, and $A$ and $B$ are computationally determined. The fit (not shown) of the thermodynamic properties is better for the entropy and free energy than for the enthalpy. This is not surprising since the logarithmic dependence is inherent in the definition of the first two properties. The fit for the enthalpy improves as $d$ increases. Less obvious is that $B$ decreases which increasing $d$ for the entropy and decreases with increasing $d$ for both the free energy and enthalpy. Also, for $d=1$, the enthalpy does not have any $N$ dependence. 


\section{Conclusions}

We have determined the asymptotic behavior several properties of clusters of hypercubes in $d$-dimensional space. This includes six topological indices, the graph energy and thermodynamic properties such as entropy, free energy and enthalpy. For the case of $L=1$, our calculations are in agreement with previously published formulas for four topological indices. The graph energy for $d=2$ is close to the value determined mathematically, and we add limits for several more dimensions. The thermodynamic properties have large $N$ logarithmic behavior in agreement with the definitions.

\section{References}

[1] F. Harary, J.P. Hayes, H.Y. Wu, A Survey of the Theory of Hypercube Graphs, Comput. Math. Applic. 15(4) (1988) 277-289.

[2] M. Abd-El-Barr, F. Gebali, Reliability analysis and fault tolerance for hypercube multicomputer networks, Information Sciences 276, (2014) 295-318.

[3] J.P. Hayes, T. Mudge, Q.F. Stout, S. Colley, J. Palmer, A Microprocessor-based Hypercube Supercomputer, IEEE Micro 6(5), (1986) 6-17.

[4] B.K. Teo, N.J.A. Sloane, Magic numbers in polygonal and polyhedral clusters, Inorg. Chem. 24, (1985) 4545-4558.

[5] J.M. Montejano-Carrizales, F. Aguilera-Granja, J.L. Moran-Lopez, Direct enumeration of the geometrical characteristics of clusters, NanoStructured Mater. 8(3), (1997) 269-287.

[6] Y. Xia, Y Xiong, B. Lim, S.E. Skrabalak, Shape-Controlled Synthesis of Metal Nanocrystals: Simple Chemistry Meets Complex Physics?, Angew. Chem. Int. Ed. 48, (2009), 60-103. [7] D.J. Norris, A.L. Efros, S.C. Erwin, Doped Nanocrystals, Science 319, (2008), 1776-1779.

[8] H. Wiener, Structural determination of paraffin boiling points, J. Am. Chem. Soc. 69, (1947) 7-20.

[9] H. Hosoya, Topological index. A newly proposed quantity characterizing the topological nature of structural isomers of saturated hydrocarbons, Bull. Chem. Soc. Japan 44, (1971) $2332-2339$.

[10] P. Schwerdtfeger, L. Wirz, J. Avery, Program fullerene: a software package for constructing and analyzing structures of regular fullerenes, J. Comput. Chem. 34, (2013) 1508-1526. [11] F.H. Kaatz, A. Bultheel, Informational thermodynamic model for nanostructures, J. Math. Chem. 52(6), (2014) 1563-1575.

[12] F.H. Kaatz, A. Bultheel, Topological Indices for Nanoclusters, Comput. Mater. Sci., (2014).

[13] O. Ori, F. Cataldo, D. Vukicevic, A. Graovac, Wiener Way to Dimensionality, Iranian J. Math. Chem. 1(2) (2010) 5-15.

[14] W. Yan, Z. Zhang, Asymptotic Energy of Lattices, Physica A 388, (2009) 1463-1471. 
[15] R. Albert, A.L. Barabasi, Statistical mechanics of complex networks, Rev. Mod. Phys. 74, (2002) 47-97.

[16] J. Park, M.E.J. Newman, Statistical mechanics of networks, Phys. Rev. B. 70, (2004) 066117.

[17] E. Estrada, N. Hatano, Statistical-mechanical approach to subgraph centrality in complex networks, Chem. Phys. Lett. 439, (2007) 247-251.

[18] W.R. Müller, K. Szymanski, J.V. Knop, N. Trinajstic, An algorithm for construction of the molecular distance matrix, J. Comput. Chem. 8(2), (1987) 170-173.

[19] B. Zhou, N. Trinajstic, Maximum eigenvalues of the reciprocal distance matrix and the reverse Wiener matrix, Inter. J. Quan. Chem. 108, (2008) 858-864.

[20] I. Gutman, A formula for the Wiener number of trees and its extension to graphs containing cycles, Graph Theory Notes New York 27, (1994) 9-15.

[21] P.W. Fowler, G. Caporossi, P. Hansen, Distance matrices, Wiener indices, and related invariants of fullerenes, J. Phys. Chem. A 105, (2001) 6232-6242.

[22] P.V. Khadikar, N.V. Deshpande, P.P. Kale, A. Dobrynin, I. Gutman, G. Domotor, The Szeged index and an analogy with the Wiener index, J. Chem. Inf. Comput. Sci. 35, (1995) 547-550.

[23] S. Klavzar, M.J. Nadjafi-Arani, Wiener index versus Szeged index in networks, Discr. Appl. Math. 161, (2013) 1150-1153.

[24] A.T. Balaban, Highly discriminating distance-based topological index, Chem. Phys. Lett. 89(5), (1982) 399-404.

[25] A.T. Balaban, Topological indices based on topological distances in molecular graphs, Pure Appl. Chem. 55(2), (1983) 199-206.

[26] A.T. Balaban, D. Mills, O. Ivanciuc, S.C. Basak, Reverse Wiener indices, Croat. Chem. Acta 73(4), (2001) 923-941.

[27] I. Gutman, B. Mohar, The quasi-Wiener and the Kirchhoff indices coincide, J. Chem. Inf. Comput. Sci. 36, (1996) 982-985.

[28] S.E. Massen and C.P. Panos, Universal property of the information entropy in atoms, nuclei and atomic clusters Phys. Lett. A, 26 (1998) 530-533.

[29] A. Graovac, T Pisanski, On the Wiener Index of a Graph, J. Math. Chem. 8, (1991) 53-62.

[30] M.R. Darafsheh, Computation of Topological Indices of Some Graphs, Acta Appl. Math. 110, (2010) 1225-1235.

[31] M. Ghorbani, Remarks on the Balaban Index, Serdica J. Comput. 7(1), (2013) 25-34.

[32] J. Liu, J. Cao, X.F. Pan, A. Elaiw, The Kirchhoff Index of Hypercubes and Related Complex Networks, Discr. Dynamics in Nature and Society 2013, Art. ID 543189 (2013) $1-7$.

[33] S. Daneshvar, G. Izbirak, M.M. Kaleibar, Topological Indices of Hypercubes, J. Basic. Appl. Res. 2(11), (2012) 11501-11505. 